\title{
Clinical and Immunological Characterization of Combined Immunodeficiency Due to TFRC Mutation in Eight Patients
}

\author{
Amal H. Aljohani ${ }^{1,2}$ (D) Hamoud Al-Mousa ${ }^{1,3} \cdot$ Rand Arnaout $^{1,3} \cdot$ Hasan Al-Dhekri ${ }^{1} \cdot$ Reem Mohammed $^{1} \cdot$ \\ Zobaida Alsum $^{4} \cdot$ Manal Nicolas-Jilwan $^{5} \cdot$ Fayhan Alrogi $^{6} \cdot$ Saleh Al-Muhsen ${ }^{1,4}$ • Anas M. Alazami ${ }^{7,8}$ • Bandar Al-Saud ${ }^{1,3}$
}

Received: 23 June 2020 / Accepted: 19 August 2020 / Published online: 27 August 2020

(C) Springer Science+Business Media, LLC, part of Springer Nature 2020

\begin{abstract}
Purpose Combined immunodeficiency (CID), due to mutations in TFRC gene that encodes the transferrin receptors (TfR1), is a rare monogenic disorder. In this study, we further characterize the clinical and immunological phenotypes in a cohort of eight patients.

Methods A retrospective review of clinical and immunological features of patients diagnosed with a TFRC gene mutation between 2015 and 2019 in three tertiary centers.

Results Eight patients from six unrelated families were enrolled. The patients had a median age of 7 years (4-32 years). All patients presented with recurrent sinopulmonary infections, chronic diarrhea, and failure to thrive in early life. Less common features were skin abscesses, conjunctivitis, global developmental delay, optic nerve atrophy, vitiligo, multinodular goiter, and hemophagocytic lymphohistiocytosis-like symptoms. All patients had intermittent neutropenia and $87 \%$ of the patients had recurrent thrombocytopenia. Anemia was found in $62 \%$. All patients had hypogammaglobinemia and one had a persistent high IgM level. All patients had impaired function of T cells. The same homozygous missense mutation c.58T>C:p.Y20H, in the TFRC gene, was detected in all patients. Stem cell transplantation from matched donors was successful in two patients. Five patients did not receive stem cell transplantation, and they are on prophylactic treatment. One patient died due to severe sepsis and neurological complications.
\end{abstract}

Conclusion This report provides a large cohort with a long follow up of patients with this disease. Our cohort showed variable disease severity.

Keywords Primary immune deficiency · combined immunodeficiency · a mutation in TFRC · TfR1 · CD71

\section{Introduction}

Transferrin receptor 1 (TfR1) is a cell surface receptor that is necessary for cellular iron uptake, by the process of receptor-

Amal H. Aljohani

ahjohani@taibahu.edu.sa

$\triangle$ Bandar Al-Saud

balsaud@kfshrc.edu.sa

1 Section of Pediatric Allergy and Immunology, Department of Pediatrics, King Faisal Specialist Hospital \& Research Center, MBC-58, P.O. Box 3354, Riyadh 11211, Saudi Arabia

2 Department of Pediatrics, College of Medicine, Taibah University, Madinah, Saudi Arabia

3 Colleges of Medicine, Alfaisal University, Riyadh, Saudi Arabia mediated endocytosis. This receptor is present in all vertebrates and is required for erythropoiesis and neurologic development $[1,2]$. It acts as an essential receptor that mediates the control of iron metabolism [3]. The molecular weight of the

Department of Pediatrics, College of Medicine, King Saud University, Riyadh, Saudi Arabia

5 Department of Radiology, King Faisal Specialist Hospital \& Research Center, Riyadh, Saudi Arabia

6 Department of Pediatrics, King Abdullah Specialist Children's Hospital, King Abdullah International Medical Research Center, King Saud bin Abdulaziz University for Health Sciences, Ministry of National Guard Health Affairs, Riyadh, Saudi Arabia

7 Department of Genetics, King Faisal Specialist Hospital \& Research Center, Riyadh, Saudi Arabia

8 Saudi Human Genome Program, King Abdulaziz City for Science and Technology, Riyadh, Saudi Arabia 
TfR1 homodimer is $180 \mathrm{kDa}[2,3]$. Diferric transferrin (TF) interacts with the cell surface receptor (TfR) to initiate receptor-mediated endocytosis into specialized endosomes. Then, endosomal acidification leads to iron release and $\mathrm{TfR}$ transport out of the endosome, due to the activity of divalent metal transporter 1 (DMT1). The TF and TfR complex is then recycled to the cell surface with a return to neutral $\mathrm{pH}$ that leads to a loss of transferrin affinity for its receptor. There are two ligands of TfRs, transferrin (Tf) and hemochromatosis protein (HFE); both ligands have a role in iron metabolism and regulation. HFE, which is a non-classical MHC I molecule, regulates intracellular iron loading by the modulation of $\mathrm{TfR}$ and ferritin, as its expression leads to a decrease in the level of ferritin and an increase in the level of TfR expression. This phenomena is also observed in TFRC and STEAP mutations (the transmembrane epithelial antigen protein-3) $[4,5]$. HFE forms a complex with TfR that prevents HFE degradation. TfR 1 is located near HLA class I molecules on T cells, but there is no TfR1 association with HLA class I on the surface of B cells. TfR1 and HLA class II molecules form a cluster on $T$ cells, while only a modest association was found on $\mathrm{B}$ cells [6].

A homozygous p.Tyr20His substitution in TfR 1 , which is encoded by the TFRC gene, was recently reported as a new cause of combined immunodeficiency [4]. This substitution disrupts the TfR 1 internalization motif, resulting in defective receptor endocytosis and markedly increased TfR 1 expression on the cell surface. This mutation is characterized by severe infection during childhood that leads to death if untreated. The p.Tyr20His substitution is also associated with hypogammaglobulinemia and normal lymphocyte counts, but there is impaired $\mathrm{T}$ and $\mathrm{B}$ cell function, intermittent neutropenia, and thrombocytopenia. Additionally, mild anemia with low mean corpuscular volume (MCV) has been observed in most patients who were resistant to iron supplementation. Early hematopoietic stem cell transplantation (HSCT) from matched donors leads to the resolution of clinical and laboratory abnormalities [4]. To the best of our knowledge, since the first report by Jabara et al. [4] that describes the combined immunodeficiency caused by a mutation in the TFRC gene, no other studies on the subject have been published. The extreme rarity of published reports in the literature may be due to a lack of physician awareness or the misdiagnosis of this condition as a common variable immunodeficiency or as other immunodeficiency-associated thrombocytopenia. Nevertheless, the high rate of consanguineous marriages in the Saudi population [7] and the availability of advanced molecular genetic testing provided us with the opportunity to identify and review a relatively large number of patients with combined immunodeficiency due to mutated TFRC in great detail.

\section{Methods}

\section{Clinical Data}

A retrospective chart review was performed for all patients with a mutation in TFRC who were followed up at the King Faisal Specialist Hospital and Research Centre in Riyadh (KFSHRC), the King Abdullah Specialist Children's Hospital, and the King Khalid University Hospital. After the hospitals' research advisory committee approved the study, informed consent was obtained from the patients or their parents.

\section{Immunophenotyping}

To determine the percentages and absolute counts of the lymphocyte subsets, a 1-mL peripheral blood specimen was collected from each patient. For immunofluorescent studies, CD3 FITC and CD4 PE-Cy7 antibodies were used; all monoclonal antibodies used were from BD insert attached (Becton Dickinson). Specimens were prepared, and the procedures were followed as standard methods per the manufacturer's instructions (Becton Dickinson). Specimens were then acquired and analyzed on a BD FACSCanto flow cytometer (Becton Dickinson). Specimens were then acquired on a FACSCanto II flow cytometer (Becton Dickinson) and were analyzed using BD FACSCanto clinical software [7, 8].

As previously described [9], to determine patient T lymphocyte proliferation, a 3-mL peripheral blood specimen was collected from each patient. Peripheral blood mononuclear cells were prepared by centrifugation over Ficoll-Paque and $2.5 \times 10^{5}$ mononuclear cells were cultured in $0.5 \mathrm{~mL}$ of RPMI $1640+10 \% \mathrm{AB}$ serum for 3 days in the presence of phytohemagglutinin (PHA) $(10 \mu \mathrm{g} / \mathrm{mL})$. The results of three cultures are expressed as the mean counts per minute (cpm) of thymidine incorporation [3 H] [9].

\section{Sanger Sequencing}

Genomic DNA was extracted from whole blood samples. Primers were designed to span the exon/intron boundaries with 5'-tagged M13 sequences. DNA was sequenced with BigDye Terminator.

\section{Whole Exome Sequencing}

One hundred nanograms of DNA from the index case was treated to obtain an Ion Proton AmpliSeq library. DNA was amplified in 12 separate wells using Exome Primer Pools, AmpliSeqHiFi mix (Thermo Fisher, Carlsbad, CA, USA), and 10 amplification cycles. All 12 PCR pools were combined in one well and were subjected to primer digestion and incubated with FuPa reagent (Thermo Fisher). Amplified exome 
targets were ligated with Ion P1 and Ion Xpress Barcode adapters. After purification, libraries were quantified using quantitative PCR with the Ion Library Quantification Kit (Thermo Fisher). The prepared exome library was further used for emulsion PCR on an Ion OneTouch System, and templated ion sphere particles were enriched using Ion OneTouch ES. Both procedures followed the manufacturer's instructions. The template-positive Ion PI Ion Sphere particles were processed for sequencing on the Ion Proton instrument (Thermo Fisher). Reads were mapped to UCSC hg19, and variants were identified using the Saudi Human Genome Program pipeline.

\section{Results}

\section{Clinical Features}

Eight patients, four males and four females, were included. These patients belonged to six different tribes of Arab descent (P1 and P2 are cousins; P6 and P7 are siblings). P1 data was previously published [4]. The median age was 7 years: ranging from 4 to 32 years. All patients were products of consanguineous marriages, and there was a family history of early deaths in two families (P4, P6). P4's family had one child who died at 24 months of age and another child who died at 30 months of age; both had a history of recurrent infections. Family P6 had four cases of early death: one died in utero at 24 weeks, two died in the neonatal period with unknown cause, and one died at 4 months with a chest infection (Table 1, Fig. 1). All patients in the first year of age exhibited recurrent sinopulmonary infections, chronic diarrhea, and failure to thrive. Skin abscesses were observed in two patients (P1 and $\mathrm{P} 4$ ); one patient had them in the perianal area, and the other patient had abscesses on the thigh that required incision and drainage. No oral thrush was reported. One patient (P3) had eczema during infancy, which resolved later. Two patients had developmental delays (P2 and P3). One patient (P2) had delayed speech and a persistent unsteady gate. The other patient (P3) exhibited developmental regression at 18 months of age with progressive spasticity in all limbs and vision loss that was associated with optic nerve atrophy. MRI of the brain and a metabolic workup revealed nothing abnormal. One year later, MRI of the brain revealed diffuse bilateral cortical atrophy with thinning of the intraorbital optic nerves and subdural hematoma (Fig. 2). One patient (P1) had facial nerve palsy, spina bifida occulta, and hypoplasia of the left common and internal carotid arteries.

Recurrent epistaxis and petechiae were noticed secondary to thrombocytopenia in seven patients (P1, P2, P3, P4, P6, P7, and $\mathrm{P} 8$ ), and there was a left subdural hematoma in patient $\mathrm{P} 3$. Bleeding symptoms improved in P6 and P7 when they reached adulthood. Three patients ( $\mathrm{P} 4, \mathrm{P} 6$, and $\mathrm{P} 7$ ) had bronchiectasis, and one (P6) underwent a left lower lobe basal segmentectomy at 9 years of age. Autoimmunity was present in three patients in the form of vitiligo in P6, multinodular goiter in P7, and Evan syndrome in P8. Patient P1 had hemophagocytic lymphohistiocytosis (HLH), as he developed fever and skin rash involving the mucous membrane. His laboratory workup showed high liver enzymes, pancytopenia, high ferritin, high triglycerides, and low fibrinogen with the presence of hemophagocytes in a bone marrow biopsy.

\section{Laboratory Findings}

In all patients except for two (P2 and P5), who had lymphopenia, normal lymphocyte counts were noticed. Recurrent neutropenia of mild to moderate severity was reported in six patients (P1, 4, 5, 6, 7, and 8). Sixty-two percent of patients had mild microcytic anemia with normal iron profiles; one patient (P3) had a low iron profile. Seven patients (P1, 2, 3, $4,6,7$, and 8) had thrombocytopenia. The mean platelet volume was normal in all patients (Table 2). Peripheral smear findings (P1, P2, P3, P6, and P7) confirmed microcytic anemia with few large platelets associated with reactive lymphocytes and monocytosis. Additionally, bone marrow aspirate (BMA) was collected from three patients (P1, P2, and P3), which showed hypocellularity with absent or reduced megakaryocytes. All patients had no detectable antiplatelet antibodies. Immunoglobulins ( $\operatorname{IgG}, \operatorname{IgA}$, and $\operatorname{IgM}$ ) were low in all patients except for one (P7), who had persistent high IgM levels (Table 3 ). The total number of $\mathrm{CD}^{+}$and $\mathrm{CD}^{+} \mathrm{T}$ cells was within the normal range except for one patient $(\mathrm{P} 2)$, who had persistently low lymphocyte counts. Cytotoxic $\left(\mathrm{CD}^{+}\right) \mathrm{T}$ cells were low in two patients (P2 and P5) (Table 3). B cells $\left(\mathrm{CD} 19^{+}\right)$were low in four patients (P2, P4, P6, and P7). Natural killer $\left(\mathrm{CD} 16^{+} / \mathrm{CD}^{2} 6^{+}\right)$cells were persistently low in four patients (P2, P3, P4, and P5). Naïve $\mathrm{CD}^{+}{ }^{+} \mathrm{RA}$ and memory $\mathrm{CD}^{+} \mathrm{RO}$ results were available for four patients and were normal (Table 3 ). The proliferation of peripheral blood mononuclear cells (PBMCs) in the presence of PHA was mild to moderately depressed in all patients. To arrive at a genetic diagnosis, patient DNA was obtained from whole blood and then subjected to whole exome sequencing, if there were no previously diagnosed affected siblings or relatives. Following filtering, by zygosity and allele frequency, a homozygous variant of TFRC (TFRC:NM_003234.3:c.58T>C:p.Y20H) was identified in all patients. This was subsequently confirmed by Sanger sequencing. If siblings or other relatives were known to carry the TFRC variant, patient DNA was assessed by direct sequencing of this mutation.

\section{Management}

Hematopoietic stem cell transplant (HSCT) was successful in two patients (P1 and P5) who had a severe phenotype; there was a good immune reconstitution and resolution of clinical 


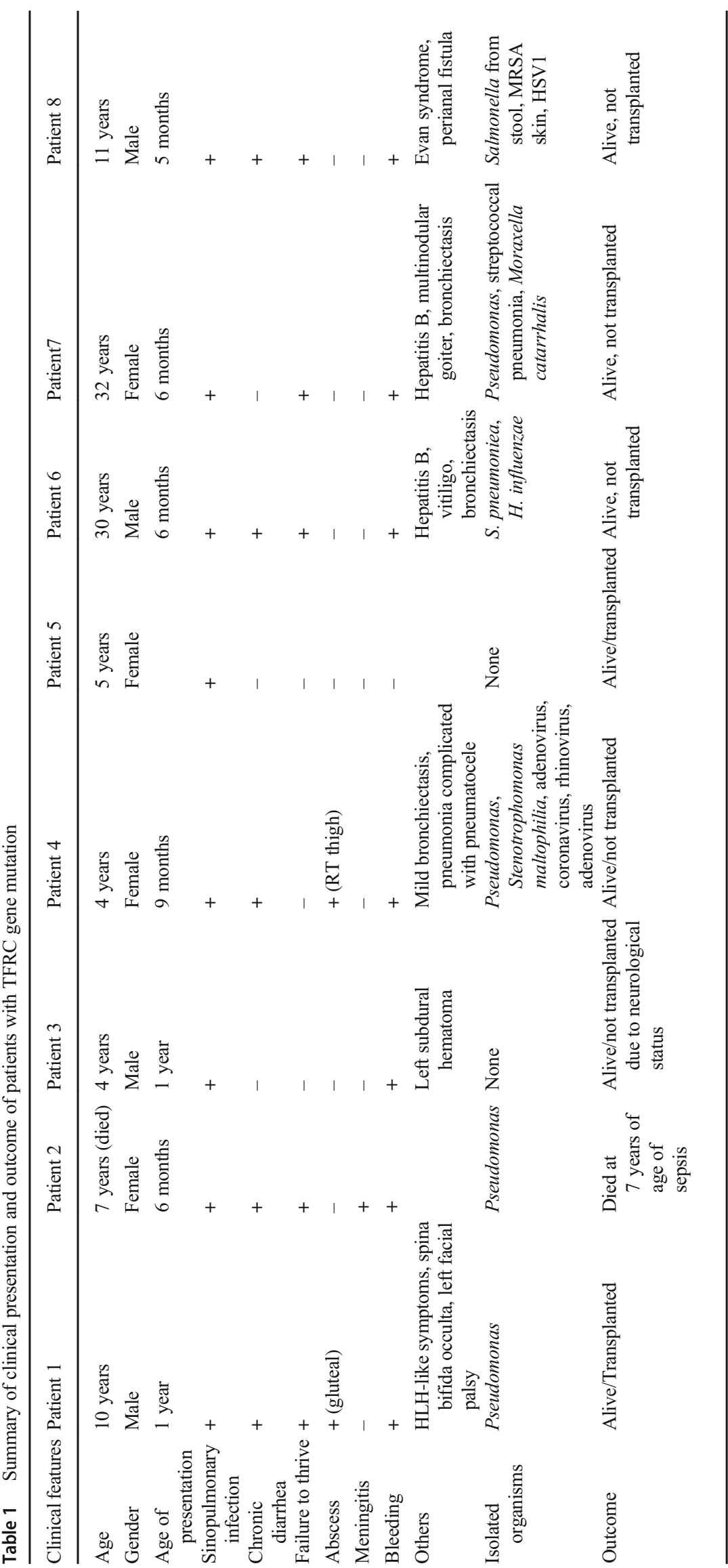


Fig. 1 Clinical manifestations, hematological, and immunological of patients with TFRC gene mutation are represented as percentages. Blue bars indicate clinical manifestations, orange bars indicate hematological findings, and green bars represent immunological results

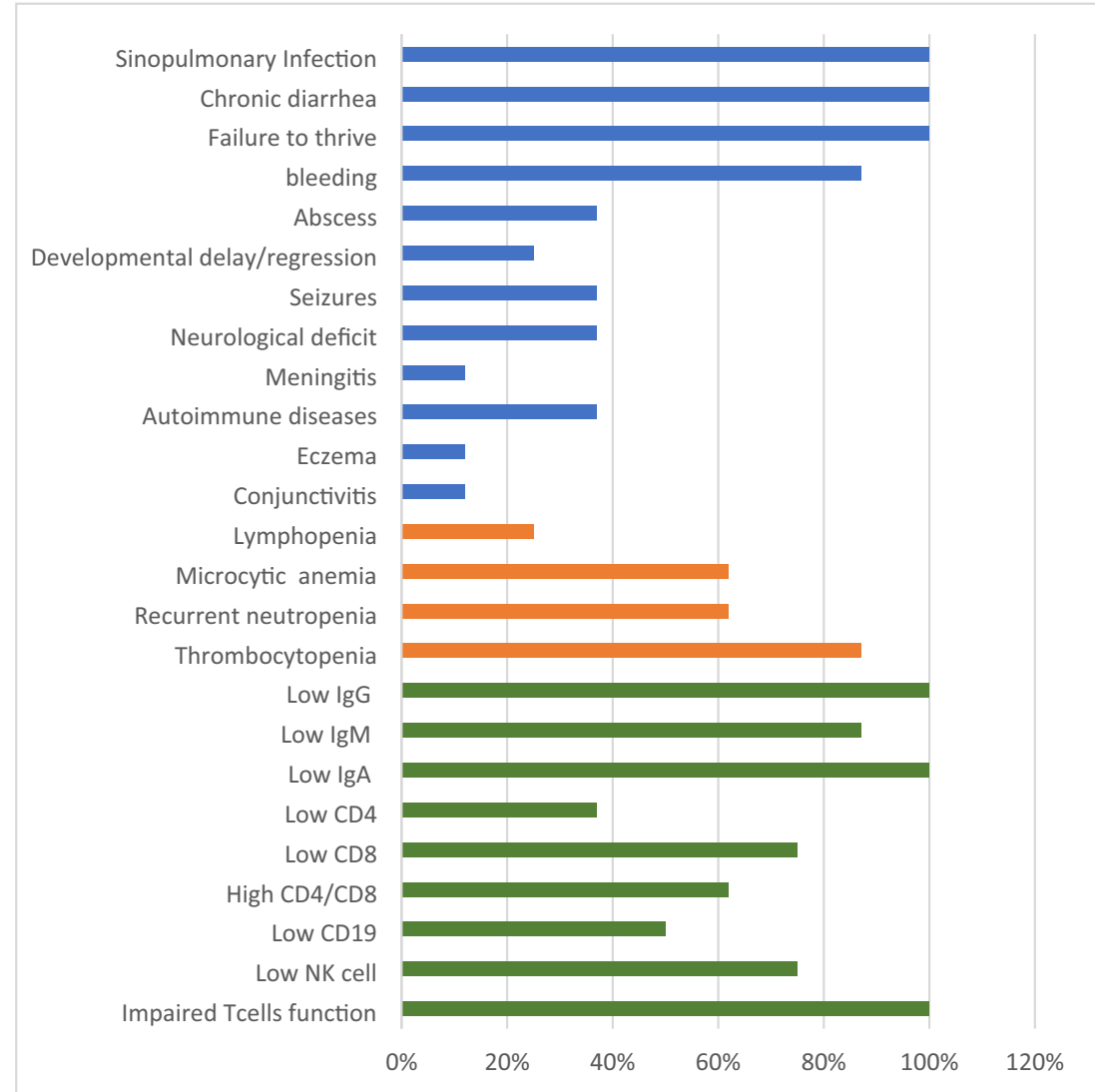

symptoms. One patient (P5) had a fully matched related donor and received myeloablative conditioning in the form of busulfan and fludarabine with methotrexate and cyclosporine as GVHD prophylaxis. The second patient (P1), who is patient (B) of the Jabara et al. paper, received a 9/10 HLA mismatched unrelated donor with myeloablative conditioning of busulfan, fludarabine, and antithymocyte globulin (ATG). The patient also received GVHD prophylaxis in the form of cyclosporine [4]. The other non-transplanted patients were administered monthly intravenous immunoglobulins and prophylactic antibiotics, and one patient (P2) died due to sepsis. Thrombocytopenia was treated with thromboplastin in three patients (P2, P3, and P8) with good response. Interestingly, the severe thrombocytopenia in two patients (P6 and P7) improved without intervention when they reached adulthood.

\section{Discussion}

Transferrin receptors (TfRs) are membrane glycoproteins that can import iron by binding transferrin (TF). It was discovered that two families had a missense mutation (Y20H) in TFRC $[2,4,10]$. Individuals with this mutation experience chronic diarrhea, recurrent infections, hypogammaglobulinemia, thrombocytopenia, and mild microcytic anemia early in their lives [4].
The two patients (P6 and P7) who survived into adulthood were labeled as having common variable immunodeficiency (CVID early in their lives). The course of their disease was complicated by bronchiectasis and thrombocytopenia; however, the severity decreased over time.

TfRs are generally needed for proliferating cells, but they are also present in the CNS, as TfR 1 appears in the brain's endothelial cells, neurons, and oligodendrocytes when the metabolically active neurons need more iron. The high respiratory rate of the brain is linked to oxidative phosphorylation in the mitochondrial respiratory chain, in which enzymes include iron as part of their prosthetic group. The observed neurological involvement in some of the patients in the form of developmental delay, milestone regression, and optic atrophy could be related to recurrent infections and prolonged hospitalization, or it could be part of the disease itself, as mitochondrial iron supply may be affected by this mutation [11-16]. One patient (P1) had "HLH"-like symptoms but no isolated pathogens and had a negative result for CMV and EBV PCR. Hemophagocytes were present in a bone marrow biopsy, and the patient responded to steroid therapy alone. As already stated, transferrin receptors act as target structures for NK cells [17]. There are a variety of cells that have detectable TfRs, including hemopoietic stem cells, as more than $90 \%$ of erythroid progenitors have detectable TfR levels [18]. In mice, TfR forms a complex with a fraction of KLRG1 (killer cell 
Fig. 2 Brain MRI at age 2 years. a Axial volumetric T1-weighted image shows diffuse bilateral cortical atrophy (thin white arrows). There is a small left frontal subdural collection (thick short arrow). b Axial FLAIR image. The subdural collection is more conspicuous (arrow) due to its high signal intensity. The hyperintense signal is suggestive of a proteinaceous content, likely evolving blood products. c Coronal fat-satured T2-weighted image of the orbits demonstrates mildly decreased caliber of the optic nerves (arrow) indicative of mild optic atrophy
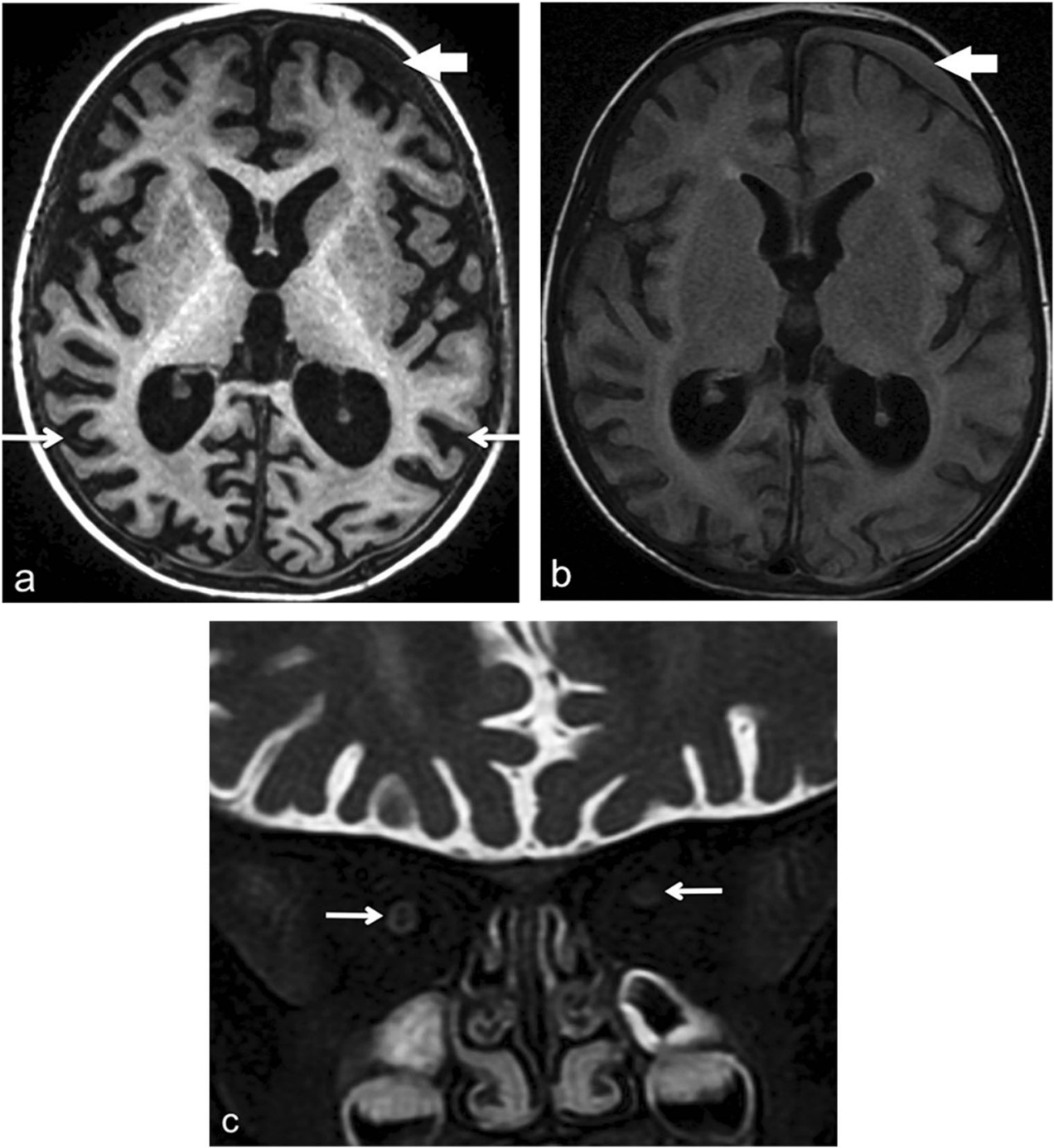

Table 2 Hematological parameters

\begin{tabular}{|c|c|c|c|c|c|c|c|c|}
\hline Parameters & Patient 1 & Patient 2 & Patient 3 & Patient 4 & Patient 5 & Patient 6 & Patient 7 & Patient 8 \\
\hline WBC $(4.30-11.30) 10 \times 9 / \mathrm{L}^{\mathrm{a}}$ & $7.97^{\mathrm{b}}$ & 6.423 & 6.95 & 6.15 & 4.25 & 6.46 & 6.26 & 8.87 \\
\hline $\begin{array}{l}\text { Neutrophil }(1.35-7.50) \\
10 \times 9 / \mathrm{L}\end{array}$ & 1.99 & 4.82 & 1.85 & 1.63 & 2.35 & 2.60 & 3.04 & 2.66 \\
\hline $\begin{array}{l}\text { Lymphocyte }(1.90-4.90) \\
10 \times 9 / \mathrm{L}\end{array}$ & 3.235 & 1.65 & 3.0 & 4.108 & 1.87 & 3.418 & 2.334 & 3.01 \\
\hline Eosinophil (0.31.0) $10 \times 9 / \mathrm{L}$ & 0.26 & 0.84 & 0.238 & 0.11 & 0.7 & 0.014 & 0.131 & 0.18 \\
\hline Hemoglobin $(110-150 \mathrm{~g} / \mathrm{L})$ & 93.61 & 91.07 & 98.34 & 108.47 & 95.5 & 137 & 111.3 & 136 \\
\hline $\operatorname{MCV}(75-95 \mathrm{fL})$ & 67.58 & 73.64 & 67.60 & 74.74 & 72.95 & 79.09 & 84.18 & 70 \\
\hline Platelets $(155-435) 10 \times 9 / \mathrm{L}$ & $\begin{array}{l}128.2 \\
\quad(1-623)^{\mathrm{c}}\end{array}$ & $\begin{array}{l}60.15 \\
\quad(14-115)\end{array}$ & $\begin{array}{l}68.48 \\
\quad(3-333)\end{array}$ & $\begin{array}{l}218.64 \\
\quad(30-530)\end{array}$ & $\begin{array}{l}218 \\
\quad(176-260)\end{array}$ & $\begin{array}{l}109.9 \\
\quad(45-221)\end{array}$ & $\begin{array}{l}241 \\
\quad(20-672)\end{array}$ & $\begin{array}{c}531 \text { (before } \\
\text { treatment } \\
1 \text { to } 5 \text { ) }\end{array}$ \\
\hline MPV (6.3-11.2 fL) & 9.4 & 8.4 & 9.82 & 10.35 & 10.05 & 10.2 & 10.47 & 8 \\
\hline
\end{tabular}

${ }^{\mathrm{a}}$ The normal values

${ }^{\mathrm{b}}$ The result at presentation

${ }^{\mathrm{c}}$ The result range for each patient 
lectin-like receptor G1) receptors, which are primarily expressed by NK cells and differentiated $\mathrm{T}$ cells at the late stage of cellular maturation or differentiation; these complexes were detected in IL-2-activated NK cells but not in resting NK cells. KLRG1 has a role in cancer immunosurveillance and preventing autoimmunity. KLRG1 may also modulate lymphocyte activation, proliferation, and responsiveness. High levels of TfRs correlate with a decrease in KLRG1-mediated inhibition and vice versa [19].

Most patients had only mild anemia, which might be explained by the existence of other mechanism for ferritin entry into the cells. As mentioned by Jabara et al., the interaction between STEAP3 and TfR 1 allows partial TfR 1 endocytosis pathway signals and thereby prevents severe anemia in patients with TFRC mutations $[4,20]$. Persistent, recurrent thrombocytopenia was detected in six patients. In these cases, the condition was severe and necessitated immediate platelet transfusion. There was no evidence of an autoimmune etiology based on the absence of platelet antibodies, hypocellular bone marrow with reduced megakaryocytes, and inadequate response to intravenous immunoglobulins, steroids, and antiCD20 treatment. There was a good response, however, to thromboplastin, which may indicate possible defects in platelet maturation. The expression of TfRs during normal platelet maturation is found in $64 \%$ of megakaryocyte precursors, but this percentage decreases until it disappears at the mature megakaryocyte stage. The expression of these receptors has been linked to the differentiation process, which is similar to other hemopoietic markers [21]. The relationship between $\mathrm{TfR}$ and platelets is still unclear, as TfR expression in platelets from patients with TFRC gene mutations has not been reported before. However, there is evidence that platelets utilize transferrin for protein synthesis and mitochondrial function. Furthermore, severe iron deficiency was reported to cause thrombocytopenia [11, 22-24]. In this gene mutation, the level of serum iron is within the average range, but the platelets' intracellular iron could also be affected by impaired TfR 1 endocytosis.

One patient (P3) had eczema during infancy and was initially suspected to have Wiskott-Aldrich syndrome (WAS). Patients with WAS and TFRC gene mutations tend to have recurrent infections and thrombocytopenia. However, patients with TFRC gene mutations are not known to have eczema, and their platelet volume is known to be normal.

Low immunoglobulin values were found in all patients. One patient (P7) had persistently high IgM. Impaired class switching has been reported in patients with TFRC gene mutations [4]. Additionally, B cell development was assessed in TfR-/- mice; the results indicate that B cell development proceeds to at least the IgM stage despite the presence of fewer IgM cells in peripheral lymphoid organs [20].

The lymphocyte markers were within normal limits, with the exception of CD19 cell, NK cell, and CD8 cell numbers 
being low compared to those of CD4 cell numbers, which led to a high CD4:CD8 ratio. This finding could be due to the role of TfR 1 in mediating important signal transduction events, as has already been reported [20,25]. As TfR stimulation leads to tyrosine phosphorylation of the TCR zeta-chain, the stimulated TCR complex increases tyrosine phosphorylation of TfR. Moreover, TfR appears to be physically associated with the TCR zeta-chain and the zeta-binding ZAP70 tyrosine kinase. The TfR/zeta complex has a role in transducing the TfRinduced signals that could amplify signals delivered by antigen binding to the TCR [25]. The lymphocyte requirement of transferrin and impaired TfR signaling role impaired lymphocyte proliferation to PHA in patients with TFRC gene mutations $[4,17]$.

To the best of our knowledge, this case series is a large cohort of patients with TFRC mutation. Our cohort showed a very early onset of disease manifestation and variable disease complications. Moreover, the survival of two patients into adulthood indicates the variable phenotypes and severity of patients with TFRC gene mutations. The disease severity and the very early onset warranted therapeutic intervention in the form of HSCT, before further complications. A multicenter study with a larger cohort and long-term follow-up is needed. Furthermore, CID due to TFRC gene mutations needs to be considered as a differential diagnosis for CVID, WAS, and HIGM syndrome.

\section{Compliance with Ethical Standards}

After the hospitals' research advisory committee approved the study, informed consent was obtained from the patients or their parents.

Conflict of Interest The authors declare that there is no conflict of interest.

\section{References}

1. Levy JE, Jin O, Fujiwara Y, Kuo F, Andrews N. Transferrin receptor is necessary for development of erythrocytes and the nervous system. Nat Genet. 1999;21(4):369.

2. Kawabata H. Transferrin and transferrin receptors update. Free Radic Biol Med. 2019;133:46-54.

3. Shen Y, Li X, Dong D, Zhang B, Xue Y, Shang P. Transferrin receptor 1 in cancer: a new sight for cancer therapy. Am J Cancer Res. 2018;8(6):916-31.

4. Jabara HH, Boyden SE, Chou J, Ramesh N, Massaad MJ, Benson $\mathrm{H}$, et al. A missense mutation in TFRC, encoding transferrin receptor 1 , causes combined immunodeficiency. Nat Genet. 2016;48(1): $74-8$.

5. Gross CN, Irrinki A, Feder JN, Enns CA. Co-trafficking of HFE, a nonclassical major histocompatibility complex class I protein, with the transferrin receptor implies a role in intracellular iron regulation. J Biol Chem. 1998;273(34):22068-74.
6. Mátyus L, Bene L, Heiligen H, Rausch J, Damjanovich S. Distinct association of transferrin receptor with HLA class I molecules on HUT-102B and JY cells. Immunol Lett. 1995;44(2-3):203-8.

7. O'Gorman MR. Role of flow cytometry in the diagnosis and monitoring of primary immunodeficiency disease. Clin Lab Med. 2007;27(3):591-626 vii.

8. Shahabuddin S, Al-Ayed IH, El-Rab MOG, Qureshi MI. Lymphocyte subset reference ranges in healthy Saudi Arabian children. Pediatr Allergy Immunol. 1998;9(1):44-8.

9. Stone KD, Feldman HA, Huisman C, Howlett C, Jabara HH, Bonilla FA. Analysis of in vitro lymphocyte proliferation as a screening tool for cellular immunodeficiency. Clin Immunol. 2009;131(1):41-9.

10. Conway AJ, Brown FC, Rank G, Kile BT, Morton CJ, Jane SM, et al. Characterization of Tfrc-mutant mice with microcytic phenotypes. Blood Adv. 2018;2(15):1914-22.

11. Chen C, Paw BH. Cellular and mitochondrial iron homeostasis in vertebrates. Biochim Biophys Acta. 2012;1823(9):1459-67.

12. Senyilmaz D, Virtue S, Xu X, Tan CY, Griffin JL, Miller AK, et al. Regulation of mitochondrial morphology and function by stearoylation of TFR1. Nature. 2015;525(7567):124-8.

13. Richardson DR, Lane DJ, Becker EM, Huang ML, Whitnall M, Suryo Rahmanto Y, et al. Mitochondrial iron trafficking and the integration of iron metabolism between the mitochondrion and cytosol. Proc Natl Acad Sci U S A. 2010;107(24):10775-82.

14. Kapnick SM, Pacheco SE, McGuire PJ. The emerging role of immune dysfunction in mitochondrial diseases as a paradigm for understanding immunometabolism. Metabolism. 2018;81:97-112.

15. Reichenbach J, Schubert R, Horvath R, Petersen J, Futterer N, Malle E, et al. Fatal neonatal-onset mitochondrial respiratory chain disease with T cell immunodeficiency. Pediatr Res. 2006;60(3): $321-6$.

16. Walker MA, Volpi S, Sims KB, Walter JE, Traggiai E. Powering the immune system: mitochondria in immune function and deficiency. J Immunol Res. 2014;2014:164309.

17. Baines MG, Lafleur FL, Holbein BE. Involvement of transferrin and transferrin receptors in human natural killer effector:target interaction. Immunol Lett. 1983;7(1):51-5.

18. Vodinelich L, Sutherland R, Schneider C, Newman R, Greaves M. Receptor for transferrin may be a "target" structure for natural killer cells. Proc Natl Acad Sci. 1983;80(3):835-9.

19. Steinle A. Transferrin' 'activation: bonding with transferrin receptors tunes KLRG 1 function. Eur J Immunol. 2014;44(6):1600-3.

20. Ned RM, Swat W, Andrews NC. Transferrin receptor 1 is differentially required in lymphocyte development. Blood. 2003;102(10): 3711-8.

21. Vinci G, Tabilio A, Deschamps J, Van Haeke D, Henri A, Guichard $\mathrm{J}$, et al. Immunological study of in vitro maturation of human megakaryocytes. Br J Haematol. 1984;56(4):589-605.

22. Karpatkin S, Garg S, Freedman M. Role of iron as a regulator of thrombopoiesis. Am J Med. 1974;57(4):521-5.

23. Beguin Y. Erythropoietin and platelet production. Haematologica. 1999;84(6):541-7.

24. Freedman ML, Karpatkin S. Requirement of iron for platelet protein synthesis. Biochem Biophys Res Commun. 1973;54(2):47581.

25. Salmerón A, Borroto A, Fresno M, Crumpton MJ, Ley SC, Alarcón B. Transferrin receptor induces tyrosine phosphorylation in T cells and is physically associated with the TCR zeta-chain. J Immunol. 1995;154(4): 1675.

Publisher's Note Springer Nature remains neutral with regard to jurisdictional claims in published maps and institutional affiliations. 